\title{
Normal Values of Diffusion Tensor Magnetic Resonance Imaging Parameters in the Cervical Spinal Cord
}

\author{
Kazuki Chagawa ${ }^{1}$, Shunka Nishijima ${ }^{2}$, Tsukasa Kanchiku ${ }^{1}$, Yasuaki Imajo ${ }^{1}$, \\ Hidenori Suzuki ${ }^{1}$, Yuichiro Yoshida ${ }^{1}$, Toshihiko Taguchi ${ }^{1}$ \\ ${ }^{1}$ Department of Orthopedic Surgery, Yamaguchi University Graduate School of Medicine, Ube, Japan \\ ${ }^{2}$ Department of Orthopedic Surgery, Sento Hill Hospital, Ube, Japan
}

\section{Study Design: Prospective study.}

Purpose: We evaluated the usefulness of diffusion tensor imaging (DTI) in diagnosing patients with cervical myelopathy by determining the accuracy of normal DTI parameter values.

Overview of Literature: DTI can visualize white matter tracts in vivo and quantify anisotropy. DTI is known to be more sensitive than conventional magnetic resonance imaging (MRI) in detecting subtle pathological changes of the spinal cord.

Methods: A total of 31 normal subjects (13 men and 18 women; age, 23-87 years; mean age, 46.0 years) were included in this study. The patients had no symptoms of myelopathy or radiculopathy. A Philips Achieva 3-Tesla MRI with SE-type Single Shot EPI was used to obtain diffusion tensor images. Apparent diffusion coefficient (ADC) and fractional anisotropy (FA) values were measured as DTI parameters on axial sections of several cervical levels. Subjects were divided into two groups: $>40$ years $(n=16)$ and $\leq 40$ years $(n=15)$. A paired t-test was used to compare significant differences between the groups. ADC and FA values were most stable on axial sections.

Results: For all subjects, mean $A D C$ and FA values were $1.06 \pm 0.09 \times 10^{-3} \mathrm{~mm}^{2} / \mathrm{sec}$ and $0.68 \pm 0.05$, respectively. $A D C$ was significantly higher in subjects $>40$ years of age than in those $\leq 40$ years. There was no significant difference in $F A$ values between the two groups. The mean ADC value was significantly higher in normal subjects $>40$ years of age than in those $\leq 40$ years.

Conclusions: It is important to consider age when evaluating cervical myelopathy by DTI.

Keywords: Diffusion tensor imaging; Reference values; Cervical spinal cord

\section{Introduction}

Diffusion tensor imaging (DTI) is a relatively new magnetic resonance imaging (MRI) technique capable of depicting structural details of the brain and spinal cord white matter. It can assist visualization of white matter tracts in vivo and quantify anisotropy [1]. DTI is computed by tensor analysis, which is a multilinear vector parameter that enables determination of the restricted anisotropic diffusion of water molecules in the structures of living organisms such as white matter nerve fibers [2].

DTI results are quantified by two primary parameters:

Received May 25, 2015; Revised May 27, 2015; Accepted May 27, 2015

Corresponding author: Kazuki Chagawa

Department of Orthopedic Surgery, Yamaguchi University Graduate School of Medicine,

1-1-1 Minami-kogushi, Ube 755-8505, Japan

Tel: +81-836-22-2268, Fax: +81-836-22-2267, E-mail: chagawa@yamaguchi-u.ac.jp 
the apparent diffusion coefficient (ADC) value, which refers to the overall diffusivity of the tissue irrespective of directional dependence, and the fractional anisotropy (FA) value, which reflects the directional dependence of the diffusion process and is expressed as a relative number ranging from 0 to 1 that increases in relation to the anisotropic diffusion within the tissue being evaluated.

Mamata et al. [3] reported that in nerve fiber impairment, the medullary sheath is destroyed and water molecules exhibit increased diffusion in the direction in which they are normally restricted (i.e., diffusion anisotropy is lost); thus, ADC increases and FA decreases. The spinal cord white matter consists of nerve fiber bundles aligned regularly from head to tail. Water molecules diffuse along the component axons resulting in high anisotropic diffusion within the spinal cord white matter, thus facilitating the usefulness of DTI in spinal cord evaluation.

Some reports have suggested the usefulness of DTI parameters for examining the cervical spinal cord $[1,4,5]$, but little has been reported on normal values of these parameters. For standard implementation of DTI as a examination method for the cervical spinal cord, it is important to study these normal values in more detail.

The aim of this study was to investigate the accuracy of normal values of DTI parameters and determine their usefulness for definitive diagnoses in patients with cervical myelopathy. We also determined if DTI could be used to objectively evaluate function in compressive cervical disease. As a first step, we calculated normal values of DTI parameters in healthy subjects.

\section{Materials and Methods}

This study was approved by the Institutional Review Board of Yamaguchi University Hospital and adhered to the tenets of the Declaration of Helsinki.

\section{Subjects}

A total of 31 healthy adults (13 men, 18 women; age, 2387 years; mean age, $46.0 \pm 18.8$ years) participated in this study. Informed consent was obtained prior to the MRI procedure. Ethics approval was obtained from the local Human Research Ethics Board. All subjects who submitted written informed consent met the exclusion criteria for MRI and exhibited no symptoms of myelopathy or radiculopathy. Subjects were divided into two groups on the basis of age-those $>40$ years old $(\mathrm{n}=16)$ and those $\leq 40$ years old ( $\mathrm{n}=15)$ - and sex (men, $\mathrm{n}=13$; women, $\mathrm{n}=18$ ). The paired $t$-test was used to identify significant differences between groups. Statistical significance was established at $p<0.05$.

\section{Imaging procedures}

A 3-T MR scanner (Philips Achieva, Philips Medical System, Eindhoven, The Netherlands) equipped with a 16-channel neurovascular coil was used to perform all examinations from 2010 to 2013. The protocol included conventional sequences to evaluate the spine and spinal cord morphology including T1 and T2. DTI data were acquired by using SE-type Single-Shot EPI sequences. The following scanning parameters were used: TE, $69 \mathrm{~ms}$; TR, 9,079 ms; number of slices, 30; interslice gap, $0 \mathrm{~mm}$; band width, $1,711.8 \mathrm{~Hz} /$ pixel; voxel size, $1.79 \times 1.42 \times 4.00 \mathrm{~mm}$; acquisition matrix, $112 \times 140$; and number of excitation, 4 . Images were acquired with $\mathrm{b}$ values of 0 and $700 \mathrm{~s} / \mathrm{mm}^{2}$. The DTI parameters, ADC and FA, were measured on axial sections of several cervical levels (C1/2-C6/7).

As shown in Fig. 1, both ADC and FA images were taken, and intraspinal ADC and FA values were measured between each vertebra from the $\mathrm{C} 1 / 2$ to $\mathrm{C} 6 / 7$ level using an axial image that gave stable values. Because DTI is incapable of capturing an image of multiple stacks simultaneously, individual slices were generated at the $\mathrm{C} 4 / 5$ level (a preferred site) as a reference (Fig. 1A). The region of interest was identified manually in the spinal axial view but did not involve the spinal edge to achieve stable values (Fig. 1B-E).

\section{Results}

$\mathrm{ADC}$ and FA values were most stable on axial sections. Fig. 2 showed the trends of ADC and FA values, respectively, in the 31 healthy subjects. The average ADC value was $1.06 \pm 0.09 \times 10^{-3} \mathrm{~mm}^{2} / \mathrm{sec}$, and the average $\mathrm{FA}$ value was $0.68 \pm 0.05$.

The trend of average ADC values and their standard deviations in the healthy subjects were shown in Fig. 3A. In general, stable values were obtained, but they tended to increase towards the lower cervical levels. As shown in Fig. 3B, a generally stable average FA value was obtained.

Next, we compared normal ADC and FA values according to age. ADC values were significantly higher 

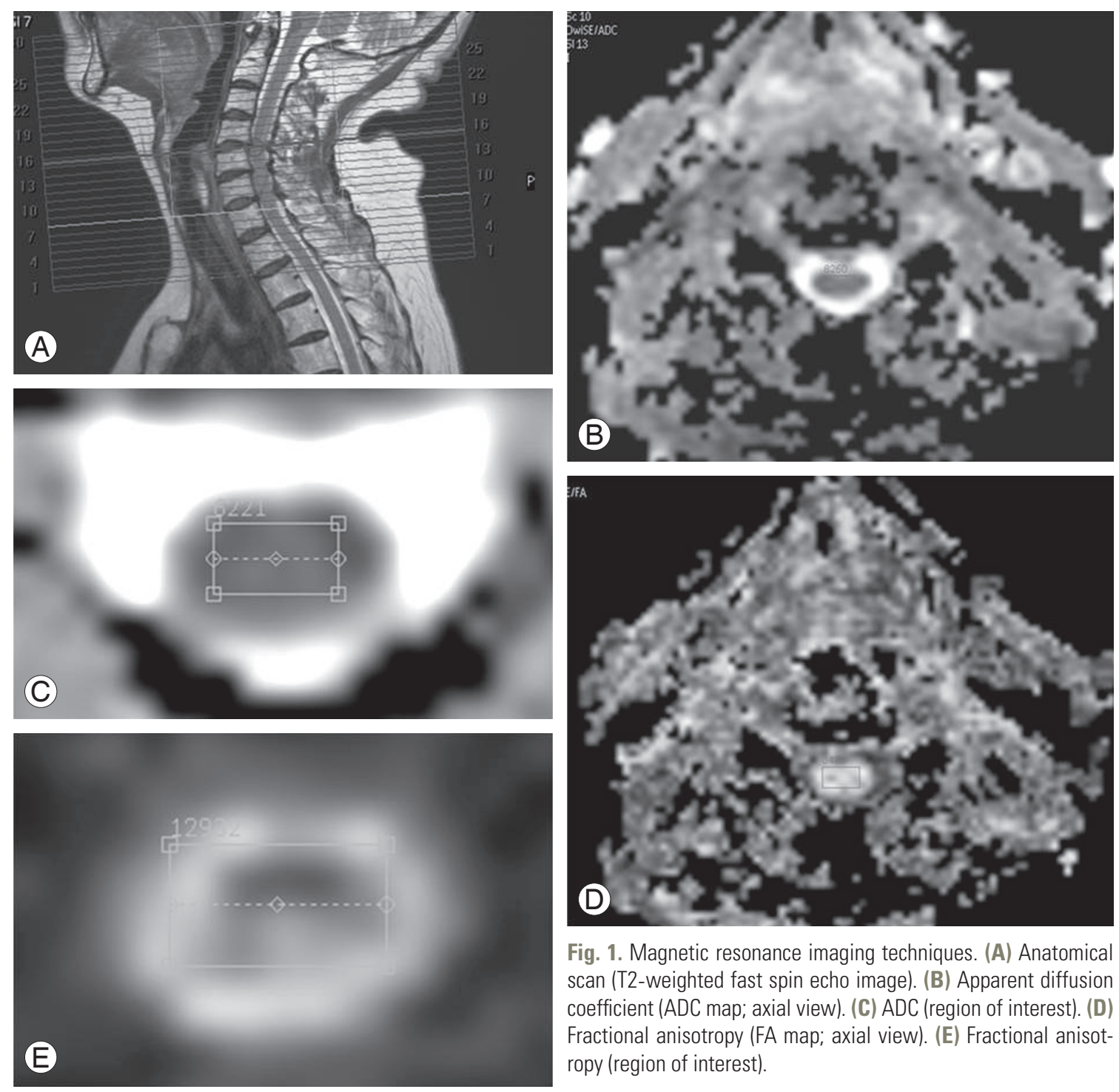

Fig. 1. Magnetic resonance imaging techniques. (A) Anatomical scan (T2-weighted fast spin echo image). (B) Apparent diffusion coefficient (ADC map; axial view). (C) ADC (region of interest). (D) Fractional anisotropy (FA map; axial view). (E) Fractional anisotropy (region of interest).
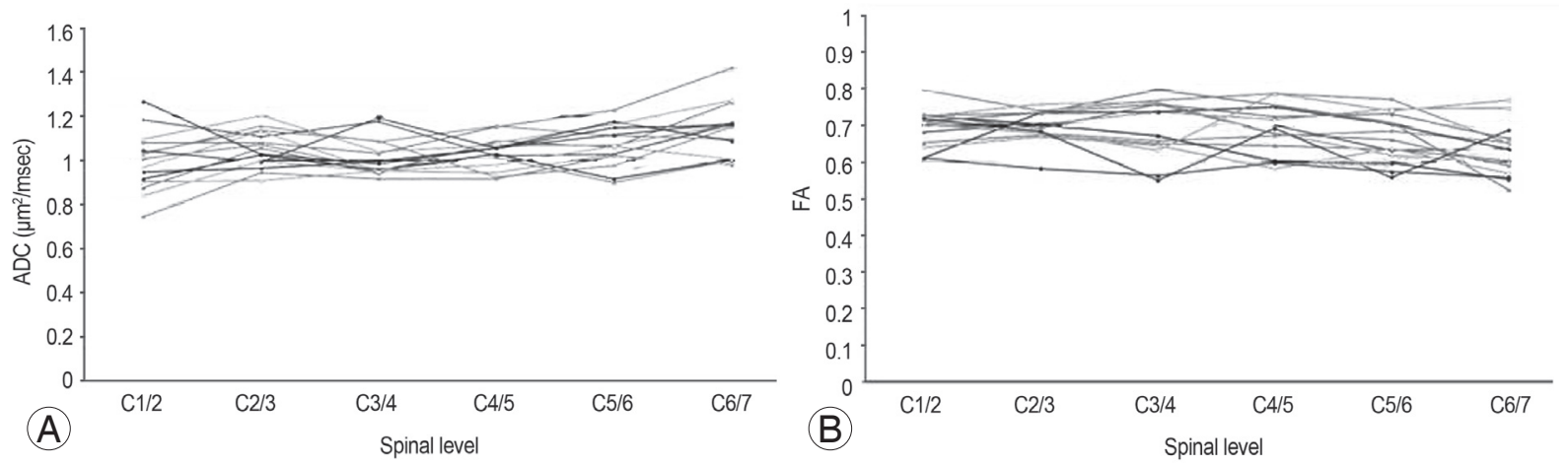

Fig. 2. (A) Apparent diffusion coefficient (ADC) in 31 healthy adults; mean $A D C=1.06 \pm 0.09 \times 10-3 \mathrm{~mm}^{2} / \mathrm{sec}$. (B) Fractional anisotropy (FA) in 31 healthy adults; mean $F A=0.68 \pm 0.05$.

in the subjects $>40$ years of age than in those $\leq 40$ years $(p=0.04)$ (Fig. 4A). Spearman rank correlation coefficient test showed that ADC was positively correlated with age $(p=0.02)$ (Fig. 4B). 

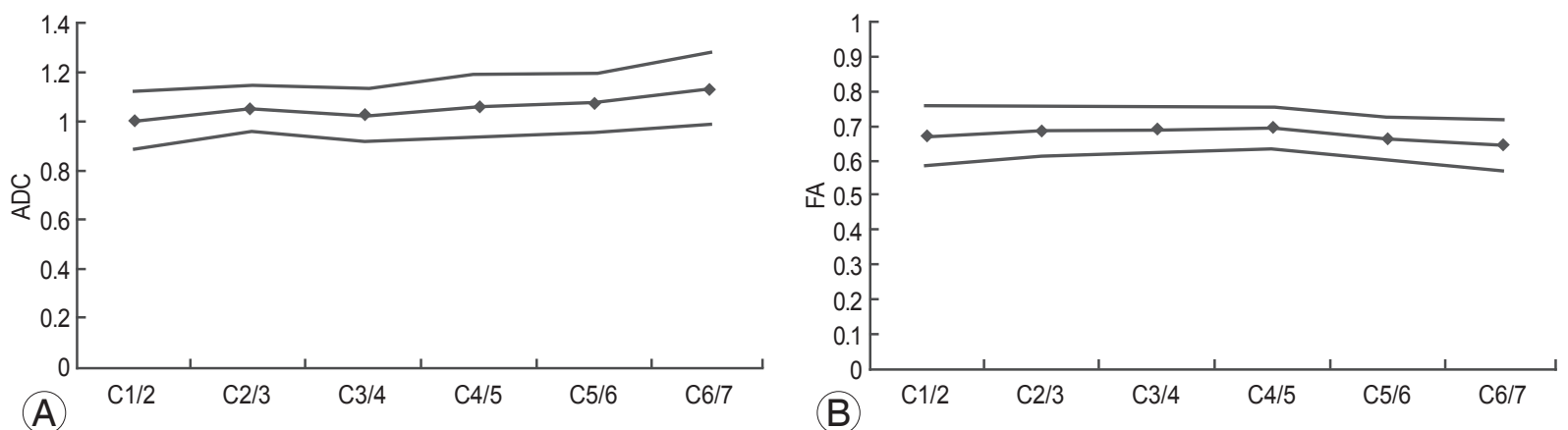

Fig. 3. (A) Apparent diffusion coefficient (ADC) (mean \pm standard deviation [SD]). (B) Fractional anisotropy (FA) (mean $\pm S D)$.

There was no significant difference in FA values among the age groups $(p=0.07)$ (Fig. $4 \mathrm{C})$. Spearman rank correlation coefficient test showed that there was a weak negative correlation between FA and age, but it was not statistically significant $(p=0.14)$ (Fig. $4 \mathrm{D})$. Pearson correlation coefficient test showed that there was a weak negative correlation between ADC and FA values. However, the correlation was not statistically significant $(p=0.07)$ (Fig. $4 \mathrm{E})$. Moreover, although the ADC value tended to be higher in women, there was no significant difference in ADC values between men and women ( $p=0.24$ ) (Fig. $4 \mathrm{~F}$ ). There was no significant difference in FA values between the two groups ( $p=0.051$ ) (Fig. $4 \mathrm{G}$ ); in addition, there was no significant difference in age between the 2 sexes (Fig. 5).

The mean percentage of ADC relative to the normal values at the $\mathrm{C} 1 / 2$ level was shown in Fig. 6A, and the mean percentage of $\mathrm{FA}$ relative to the normal $\mathrm{ADC}$ values at the C1/2 level was shown in Fig. 6B. Stable values were calculated for both parameters.

\section{Discussion}

Although some previous studies have compared parameters before and after surgery for compressive cervical disease using DTI $[1,4,5]$, few have reported on normal values. Despite the influence of the imaging environment and individual differences on average ADC or FA values in healthy subjects $[3,5]$, we obtained equivalent and stable normal values, as reported previously (Table 1) [3,6,7].

Mamata et al. [3] reported that in healthy subjects, ADC increased with age, whereas FA tended to decrease. In this study, as in previous reports, $\mathrm{ADC}$ was significantly higher in subjects $>40$ years of age; however, there was no significant difference in FA, and no difference between $\mathrm{ADC}$ and FA was found between men and women. It is expected that new trends will emerge as the number of cases increase.

Facon et al. [5] reported that a precise measurement is difficult to obtain because the value is affected by age, the measurement site, the imaging device, and the imaging method, even in a healthy spine. The relatively stable and reproducible values obtained in our study cases differed slightly from those previously reported, possibly due to the influence of these factors. To correct for individual differences, it is necessary to make comparisons with normal levels described in previous reports [8].

There are several reports on the usefulness of DTI. For example, Mamata et al. [3] reported that an increase in

Table 1. Comparison of the normal values of the ADC and FA with values in other reports

\begin{tabular}{lccc} 
Study & No. of cases & ADC $\left(\mu m^{2} / \mathrm{msec}\right.$ & FA \\
Mamata et al. [3] & 11 & range, $0.75-0.81$ & $0.66-0.75$ \\
\hline Xiangshui et al. [6] & 21 & Mean $\pm S D, 0.78 \pm 0.08$ & $0.72 \pm 0.02$ \\
\hline Takashima et al. [7] & 10 & 1.03 & 0.64 \\
Present study & 31 & $1.06 \pm 0.09$ & $0.68 \pm 0.05$ \\
\hline
\end{tabular}

ADC, apparent diffusion coefficient; FA, fractional anisotropy. 

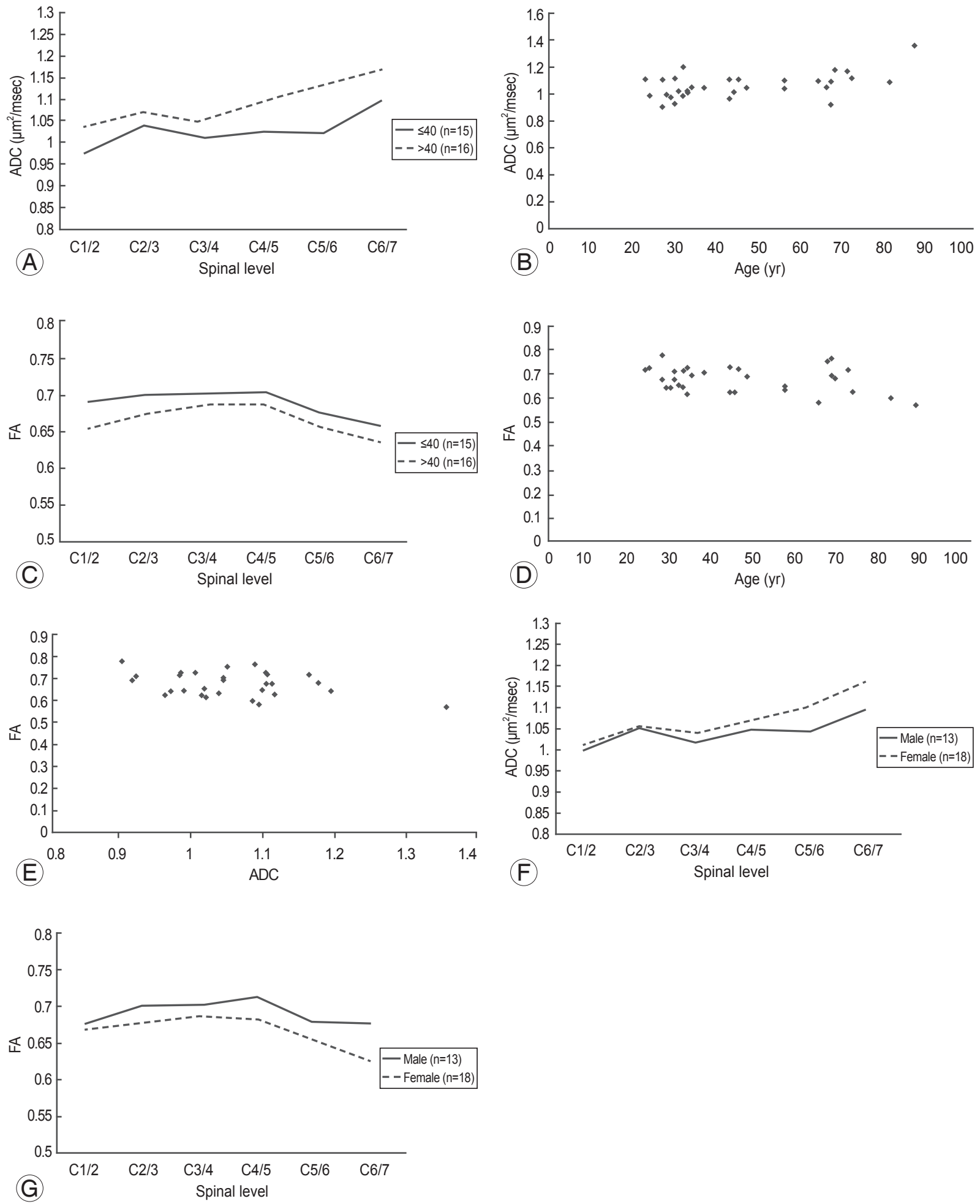

Fig. 4. (A) Apparent diffusion coefficient (ADC) was significantly higher in subjects $>40$ years of age than in those $\leq 40$ years $(p=0.04)$. (B) $A D C$ was positively correlated with age by using Spearman rank correlation coefficient test ( $p=0.02$ ). (C) Fractional anisotropy (FA) was not significantly influenced by age $(p=0.07)$. (D) Spearman's rank correlation coefficient test showed that there was a weak negative correlation between the FA and age. However, the correlation was not statistically significant ( $p=0.14)$. (E) Pearson's correlation coefficient test showed that there was a weak negative correlation between the ADC and FA. However, the correlation was not statistically significant ( $p=0.07)$. (F) There was no significant difference in the ADC between males and females ( $p=0.24)$. (G) There was no significant difference in the FA between males and females $(p=0.051)$. 


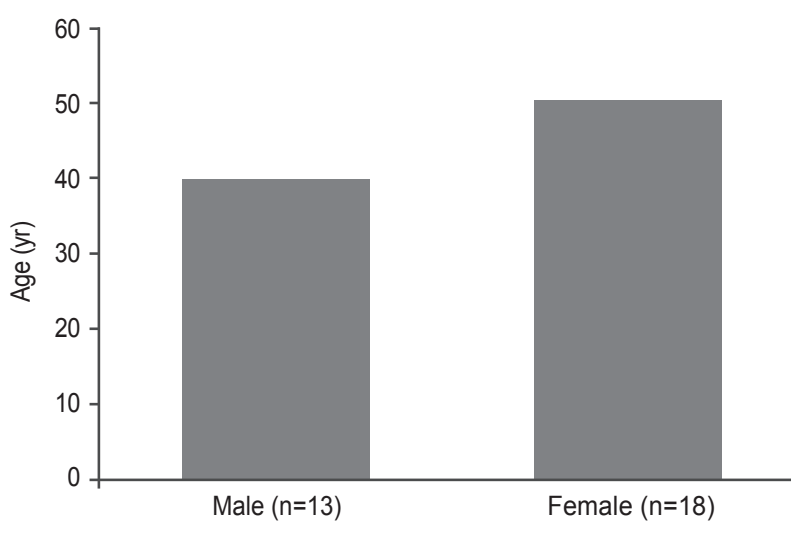

Fig. 5. There was no significant difference in the age between the two sexes.
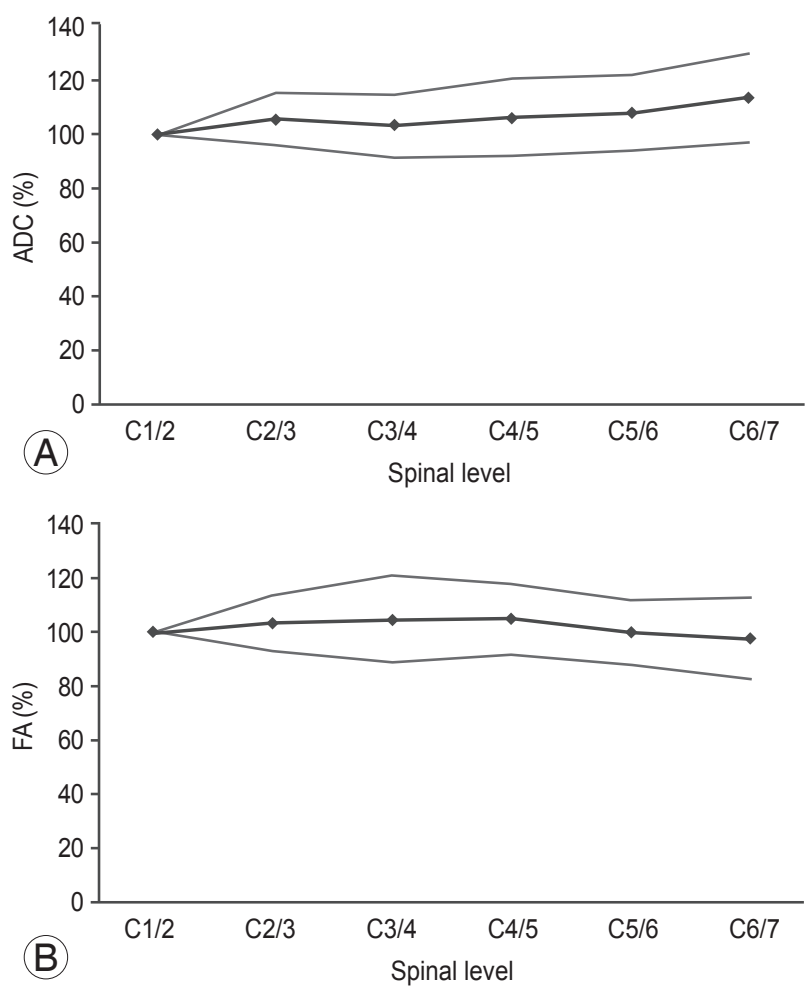

Fig. 6. (A) Percentage of apparent diffusion coefficient (ADC). (B) Percentage of fractional anisotropy (FA). Mean values indicate the percentage of the fractional anisotropy relative to the normal apparent diffusion coefficient values at the $\mathrm{C} 1 / 2$ level.

$\mathrm{ADC}$ and decrease in FA indicate the earliest stage of cervical spine disease; and Facon et al. [5] reported that a change in FA is extremely useful in detecting a spinal abnormality in the acute stage. Sei et al. [8] reported that FA and ADC correlated with several items included in the Japanese Orthopedic Association Cervical Myelopathy Evaluation Questionnaire, which suggested that these items can be used to quantify the severity of cervical spinal disease, whereas Wada et al. [1] reported that maintenance of the FA value before surgery may be an indicator of a good surgical outcome. Thus, DTI appears to be useful for the initial assessment of compressive cervical spinal disease or determining whether surgery is indicated. In addition, DTI can be used to quantify and evaluate the severity of cervical spondylotic myelopathy (CSM).

The normal ADC and FA values obtained in this study were stable and reproducible on axial views of several cervical levels and were easy to compare with abnormal values. Therefore, our findings should further contribute to the establishment of normal values. Further, the results of this study indicated that it is important to consider age and sex as DTI parameters and further suggested that measurements between each vertebral level are useful for diagnosis of impairment levels. We also found that comparing parameter values with normal levels contributed to a more accurate diagnosis of CSM. Future studies are required to confirm these results in larger numbers of healthy subjects and patients.

\section{Conclusions}

In this study, normal values of DTI parameters were identified in healthy subjects and were both reproducible and stable, which suggested that this method could be useful for patient assessment. Among the study participants, a significant difference was found only in the comparison of $\mathrm{ADC}$ by age; thus, it is important to consider age when evaluating cervical myelopathy. For diagnosis of impaired spinal levels, it is also important to consider the difference between the obtained and normal values at several levels.

\section{Conflict of Interest}

No potential conflict of interest relevant to this article was reported.

\section{References}

1. Wada A, Oda K, Kitagaki H, et al. Diffusion weighted and diffusion tensor imaging of the spinal cord. Sekitui Sekizui 2008;21:100-78.

2. Fujiyoshi K, Yamada M, Nakamura M, et al. In vivo tracing of neural tracts in the intact and injured spinal cord of marmosets by diffusion tensor tractogra- 
phy. J Neurosci 2007;27:11991-8.

3. Mamata H, Jolesz FA, Maier SE. Apparent diffusion coefficient and fractional anisotropy in spinal cord: age and cervical spondylosis-related changes. J Magn Reson Imaging 2005;22:38-43.

4. Aota Y, Niwa T, Uesugi M, Yamashita T, Inoue T, Saito T. The correlation of diffusion-weighted magnetic resonance imaging in cervical compression myelopathy with neurologic and radiologic severity. Spine (Phila Pa 1976) 2008;33:814-20.

5. Facon D, Ozanne A, Fillard P, Lepeintre JF, Tournoux-Facon C, Ducreux D. MR diffusion tensor imaging and fiber tracking in spinal cord compression.
AJNR Am J Neuroradiol 2005;26:1587-94.

6. Xiangshui M, Xiangjun C, Xiaoming Z, et al. 3 T magnetic resonance diffusion tensor imaging and fibre tracking in cervical myelopathy. Clin Radiol 2010;65:465-73.

7. Takashima H, Takebayashi Y, Yoshimoto S, Tuda H, Terashima Y, Yamashita T. Diffusion tensor imaging in cervical spinal cord by 3T MRI. J Spine Res 2011; $2 ; 3: 530$.

8. Sei A, Kitajima M, Fujimoto T, et al. MR diffusion tensor imaging in cervical myelopathy. Sekizui Kinou Shindangaku 2009;31:65-9. 